https://doi.org/10.30843/nzpp.2018.71.206

\title{
Weather and inoculum factors associated with kiwifruit bud rot
}

\author{
M. Shahjahan Kabiri, ${ }^{1, \star}$ Bethan E. Parry ${ }^{1}$, Joy L. Tyson ${ }^{2}$, Michael A. Manning ${ }^{2}$ and Robert M. Beresford ${ }^{2}$ \\ ${ }^{1}$ The New Zealand Institute for Plant \& Food Research Ltd, 412 No 1 Road, RD2 Te Puke 3182, New Zealand \\ ${ }^{2}$ The New Zealand Institute for Plant \& Food Research Ltd, Private Bag 92169, Auckland, 1142, New Zealand \\ ${ }^{*}$ Corresponding author: shahjahan.kabir@plantandfood.co.nz
}

Pseudomonas syringae pv. actinidiae biovar 3 (Psa) causes kiwifruit bacterial canker and also bud rot, which destroys developing flower buds and can become a severe problem, particularly in green-fleshed cultivars. The effects of weather and inoculum factors on bud-rot development were investigated. Experiments were conducted on two green kiwifruit cultivars: Actinidia chinensis var. deliciosa 'Hayward' and $A$. chinensis var. chinensis $\times$ A. chinensis var. deliciosa 'Zesh004' (known as Green14), at four sites for two consecutive years. Temperature and rainfall were recorded from bud burst to flowering and bud-rot incidence was monitored from approximately two weeks after flower bud appearance until flowering. Correlations between weather parameters and final bud-rot incidence, and between initial bud-rot and final bud-rot incidence were investigated. There was no significant association between temperature and final bud-rot incidence, but total rainfall and number of days of rain were positively correlated with final bud-rot incidence. Initial bud-rot incidence showed the strongest correlation with final bud-rot incidence and appeared to be the main factor that contributed to bud-rot.

https://doi.org/10.30843/nzpp.2018.71.218

\section{Propidium monoazide combined with qPCR to differentiate live and dead conidia of Neofabraea actinidiae}

\author{
Lucia Ramos $^{1, \star}$, I.P. Shamini Pushparajah ${ }^{1}$, M. Shahjahan Kabir ${ }^{2}$, Bethan E. Parry ${ }^{2}$ and Kerry R. Everett ${ }^{1}$ \\ ${ }^{1}$ The New Zealand Institute for Plant \& Food Research Ltd, Private Bag 92169, Auckland 1142, New Zealand \\ ${ }^{2}$ The New Zealand Institute for Plant \& Food Research Ltd, 412 No 1 Road, RD2 Te Puke 3182, New Zealand \\ ${ }^{*}$ Corresponding author: Lucia.Ramos@plantandfood.co.nz
}

Neofabraea actinidiae can occasionally cause post-harvest rot in kiwifruit. Quantitative polymerase chain reaction (qPCR) analysis represents a feasible and accurate option for identifying and quantifying this rot but is limited because qPCR results do not differentiate live and dead conidia. Propidium monoazide (PMA) is a photoreactive dye that penetrates into the damaged cell-wall membranes of dead conidia binding to the DNA and thus suppressing its amplification by qPCR. A commercial kit containing PMA was trialled for differentiating between live and dead N. actinidiae conidia. The most suitable conditions were $1 \mu \mathrm{M}$ PMA with 10 min light emitting diode (LED) exposure, and could clearly distinguish high concentrations of live from similar concentrations of dead conidia when tested separately and as a mixture. Low concentrations of live N. actinidiae conidia could be distinguished from dead ones when tested separately, but not as a mixture. Additional work is needed to optimise the effectiveness of the PMA binding and apply this concept in the orchard. 